\title{
An Efficient and Simple Aminobenzannulation Reaction: Pyrrolidine as a Trigger for the Synthesis of 1-Amino-Acridines.
}

\author{
Philippe Belmont,* Tahar Belhadj \\ Université Claude Bernard, Lyon I. UMR CNRS 5181, Méthodologie de Synthèse \\ et Molécules Bioactives. Bâtiment CPE, 43 boulevard du 11 Novembre 1918, \\ 69622 Villeurbanne cedex, France.
}

\section{Supporting Information-1}

(contains experimental information for new compounds reported in this paper) 


\subsection{Experimental protocols and general methods}

Melting points were determined on a Büchi B-540 apparatus. IR spectra were recorded on an FTIR spectrometer Perkin Elmer 1720, as thin films or directly with solids. ${ }^{1} \mathrm{H}$ and ${ }^{13} \mathrm{C}$ NMR spectra were recorded respectively at 300 and $75 \mathrm{MHz}$ in $\mathrm{CDCl}_{3}$ solutions on a ALS300 Brüker instrument. Chemical shifts are reported in ppm on the $\delta$ scale. Multiplicities are described as s (singlet), d (doublet), dd, ddd, etc. (doublet of doublets, doublet of doublets of doublets, etc.), $t$ (triplet), q (quartet), m (multiplet), and further qualified as app (apparent), b (broad), c (complex); coupling constants, $J$, are reported in Hz. Low and high-resolution mass spectra $(\mathrm{m} / \mathrm{z})$ were measured on a Thermo-Finnigan Mat 95XL spectrometer, with different techniques: Chemical Ionization mode (CI, isobutane as the reagent gas) or LSIMS (Liquid Secondary Ion Mass Spectrometry). Low resolution was also performed with ESI (ElectroSpray Ionization). Mass Analysis were made by Ms L. Rousset-Arzel and Dr. D. Bouchu. Flash column chromatography purifications were performed with Geduran ${ }^{\circledR}$ Si 60 from Merck (particle size 60-200 $\mu \mathrm{m}$ ), and PTLC on Analtech $1000 \mu \mathrm{m}$ or $250 \mu \mathrm{m}$.

When necessary, reactions were performed under argon atmosphere and in dried flasks. THF was freshly distilled from sodium benzophenone ketyl under nitrogen and $\mathrm{CH}_{2} \mathrm{Cl}_{2}$ was distilled from $\mathrm{CaH}_{2}$.

Method A (Table 1): In hexane $(0.25 \mathrm{~mL})$ was added, pyrrolidine or piperidine (2.94 mmol, 8 equiv) and $\mathrm{TiCl}_{4}\left(1 \mathrm{M}\right.$ in $\mathrm{CH}_{2} \mathrm{Cl}_{2}$, slow addition of $0.26 \mathrm{~mL}, 0.026 \mathrm{mmol}, 0.7$ equiv) under argon at $0-5{ }^{\circ} \mathrm{C}$. After $25 \mathrm{~min}$ at this temperature compound 3a (100 $\mathrm{mg}, 0.37 \mathrm{mmol}, 1$ equiv) was added, the mixture became yellow-orange. After $20 \mathrm{~min}$, the mixture was stirred 25 minutes at room temperature. After dilution in dry toluene $(50.0 \mathrm{~mL})$ and addition of $\mathrm{PtCl}_{2}(29 \mathrm{mg}, 0.11$ mmol, $30 \mathrm{~mol} \%$ ) it was stirred $17 \mathrm{~h}$ at $100{ }^{\circ} \mathrm{C}$. The reaction was quenched by addition of EtOAc and water. The organic layer was washed with water and brine, dried on $\mathrm{Na}_{2} \mathrm{SO}_{4}$, and then further purification gave the desired compounds, $4 \mathbf{a}$ and $\mathbf{4 b}$.

Method B (Table 1): In dry $\mathrm{CH}_{2} \mathrm{Cl}_{2}$ and under argon was added, 3a (1 equiv, 0.04-0.08 $\mathrm{M}$ in $\mathrm{CH}_{2} \mathrm{Cl}_{2}$ ), the secondary amine (3 equiv), $4 \AA$ molecular sieves ( 3 wt equiv) and, only for $\mathbf{4 a}$, neutral $\mathrm{Al}_{2} \mathrm{O}_{3}$ (1 wt equiv). The mixture was stirred at r.t. for 3-5 $\mathrm{h}$ until disappearance of the starting material (conversion to the enamine), then $\mathrm{PtCl}_{2}$ (15 mol\%) was added for $17 \mathrm{~h}$. After filtration of the reaction mixture and evaporation under reduced pressure, further purification gave the titled compounds $4 \mathbf{a}, 4 \mathbf{b}$ and $4 \mathbf{e}$. 
Method C (Table 1): In dry $\mathrm{CH}_{2} \mathrm{Cl}_{2}$ and under argon was added, 3a or 3b (1 equiv, 0.04-0.08M in $\mathrm{CH}_{2} \mathrm{Cl}_{2}$ ), the secondary amine (3 equiv) and $4 \AA$ molecular sieves ( 3 wt equiv). The mixture was stirred at reflux of $\mathrm{CH}_{2} \mathrm{Cl}_{2}$ for 3-4 h. After filtration of the reaction mixture and evaporation under reduced pressure, further purification gave the titled compounds $\mathbf{4 a - b}$ and $\mathbf{4 d - e}$.

Method D (Table 1): In dry $\mathrm{CH}_{2} \mathrm{Cl}_{2}$ and under argon was added, 3a (1 equiv, $0.2 \mathrm{M}$ in $\mathrm{CH}_{2} \mathrm{Cl}_{2}$ ), morpholine (6 equiv), $4 \AA$ molecular sieves ( 3 wt equiv) and neutral $\mathrm{Al}_{2} \mathrm{O}_{3}$ ( 1 wt equiv). The mixture was stirred at reflux of $\mathrm{CH}_{2} \mathrm{Cl}_{2}$ for $15 \mathrm{~h}$. After filtration of the reaction mixture and evaporation under reduced pressure, further purification gave the titled compound $\mathbf{4 c}$.

\subsection{Compounds 1a-b, 2a-b, 3a-b, 4a-e}

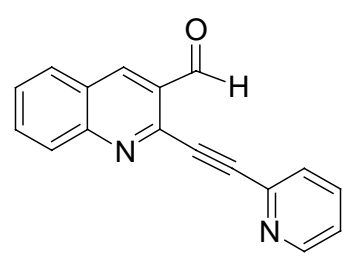

\section{2-Pyridin-2-ylethynyl-quinoline-3-carbaldehyde (1a)}

To a stirred solution of 2-chloro-3-quinoline carboxaldehyde (1.4 g, 7.30 mmol, 1 equiv) in anhydrous DMF (13.0 mL) and anhydrous THF (5.0 $\mathrm{mL}$ ) were added $\mathrm{PdCl}_{2}\left(\mathrm{PPh}_{3}\right)_{2}$ (256 mg, $0.365 \mathrm{mmol}, 0.05$ equiv), $\mathrm{Et}_{3} \mathrm{~N}$ (3.1 mL, $30.00 \mathrm{mmol}, 4.15$ equiv), $\mathrm{CuI}$ (70.0 $\mathrm{mg}, 0.365 \mathrm{mmol}, 0.05$ equiv) and 2-ethynylpyridine ( $0.82 \mathrm{~mL}, 8.07 \mathrm{mmol}, 1.11$ equiv) at room temperature. The mixture was stirred overnight and quenched by addition of EtOAc. Filtration on celite, evaporation of THF and DMF under reduced pressure and purification by flash chromatography on silica (using gradient of elution cyclohexane/EtOAc, 1/1 then 100\% EtOAc) afforded 78\% of desired product 1a as a brownish solid (1.48g, 5.73mmol). mp 167-168 ${ }^{\circ} \mathrm{C} ; \mathrm{R}_{f}$ (cyclohexane/EtOAc, 1/1): 0.30; IR (neat) 3047, 2858, 1690, 1578, $1463 \mathrm{~cm}^{-1}$; ${ }^{1} \mathrm{H}$ NMR (300 MHz, $\mathrm{CDCl}_{3}, \delta$ ): 10.86 (s, 1H), 8.80 (s, 1H), 8.72 (ddd, $J=5.1 \mathrm{~Hz}, \mathrm{~J}=2.1 \mathrm{~Hz}, J=0.6 \mathrm{~Hz}, 1 \mathrm{H}), 8.21(\mathrm{bd}, J=7.5 \mathrm{~Hz}, 1 \mathrm{H}), 8.01(\mathrm{bd}, 1 \mathrm{H}, \mathrm{J}=8.1 \mathrm{~Hz}$, 1H), 7.91 (bdd, $J=7.5 \mathrm{~Hz}, \mathrm{~J}=1.8 \mathrm{~Hz}, 1 \mathrm{H}$ ), 7.81-7.73 (m, 2H), 7.68 (ddd, $J=8.1 \mathrm{~Hz}, J=6.9 \mathrm{~Hz}$, $J=1.0 \mathrm{~Hz}, 1 \mathrm{H}), 7.37(\mathrm{ddd}, J=7.5 \mathrm{~Hz}, J=8.4 \mathrm{~Hz}, J=1.0 \mathrm{~Hz}, 1 \mathrm{H}) ;{ }^{13} \mathrm{C} \mathrm{NMR}\left(75 \mathrm{MHz}, \mathrm{CDCl}_{3}\right.$,

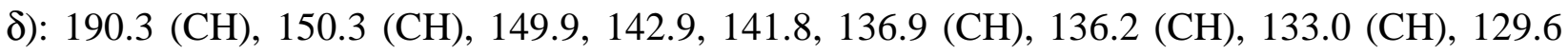
$(\mathrm{CH}), 129.3(\mathrm{CH}), 128.9,128.4(\mathrm{CH}), 127.9(\mathrm{CH}), 126.5,123.9(\mathrm{CH}), 93.4,84.2$ ppm; HRMS-CI $(\mathrm{m} / z)$ calcd for $\left[\mathrm{C}_{17} \mathrm{H}_{10} \mathrm{~N}_{2} \mathrm{O}+\mathrm{H}\right]^{+}: 259.0871$; Found: 259.0872. 


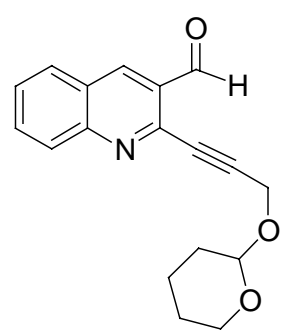

2-[3-(Tetrahydro-pyran-2-yloxy)-prop-1-ynyl]-quinoline-3-carbaldehyde (1b)

To a stirred solution of 2-chloro-3-quinoline carboxaldehyde $(1.0 \mathrm{~g}, 5.20$ mmol, 1 equiv) in anhydrous DMF $(5.0 \mathrm{~mL})$ and anhydrous THF $(10.0 \mathrm{~mL})$ were added tetrahydro-2-(2-propynyloxy)-2H-pyran $(0.90 \mathrm{~mL}, 6.24 \mathrm{mmol}$, 1.2 equiv), $\mathrm{Et}_{3} \mathrm{~N}$ (1.09 mL, $7.8 \mathrm{mmol}, 1.5$ equiv), $\mathrm{CuI}(300 \mathrm{mg}, 1.56 \mathrm{mmol}, 0.3$ equiv) and $\mathrm{PdCl}_{2}\left(\mathrm{PPh}_{3}\right)_{2}$ (256 mg, $0.36 \mathrm{mmol}, 0.07$ equiv), at room temperature. The mixture was stirred for $48 \mathrm{~h}$ and the volatiles were evaporated under reduced pressure. Addition of cyclohexane/EtOAc $(8 / 2)$ to the mixture produced two crops $(500 \mathrm{mg})$ of $\mathbf{1 b}$ and purification by flash chromatography on silica of the residue (cyclohexane/EtOAc, 8/2) afforded $1.0 \mathrm{~g}$ of $\mathbf{1 b}$. The overall yield is $97 \%$ of a light brown solid $\left(1.5 \mathrm{~g}, 5.08 \mathrm{mmol}\right.$ ). ). $\mathrm{mp} 77-79^{\circ} \mathrm{C} ; \mathrm{R}_{f}$ (cyclohexane/EtOAc, 8/2): 0.38; IR (neat) 2941, 2851, 1695, 1583,1490, 1435, 1119, 1014, $971 \mathrm{~cm}^{-1} ;{ }^{1} \mathrm{H}$ NMR (300 MHz, $\mathrm{CDCl}_{3}, \delta$ ): $10.67(\mathrm{~s}, 1 \mathrm{H}), 8.72(\mathrm{~s}, 1 \mathrm{H}), 8.12(\mathrm{bd}, J=8.48 \mathrm{~Hz}, 1 \mathrm{H}), 7.95(\mathrm{bd}, J=8.29 \mathrm{~Hz}, 1 \mathrm{H}), 7.85$ (ddd, $J=$ $7.4 \mathrm{~Hz}, J=7.0 \mathrm{~Hz}, J=1.1 \mathrm{~Hz}, 1 \mathrm{H}), 7.62(\mathrm{ddd}, J=7.2 \mathrm{~Hz}, J=7.0 \mathrm{~Hz}, J=0.9 \mathrm{~Hz}, 1 \mathrm{H}), 4.93(\mathrm{t}, J$ $=3.2 \mathrm{~Hz}, 1 \mathrm{H}), 4.63(\mathrm{t}, J=3.2 \mathrm{~Hz}, 2 \mathrm{H}), 3.90(\mathrm{ddd}, J=9.0 \mathrm{~Hz}, J=8.3 \mathrm{~Hz}, J=3.3 \mathrm{~Hz}, 1 \mathrm{H}), 3.60$ (m, 1H), 1.87-1.54 (m, 6H); ${ }^{13} \mathrm{C}$ NMR (75 MHz, $\left.\mathrm{CDCl}_{3}, \delta\right): 190.3,149.7,143.0,136.7(\mathrm{CH})$, $132.7(\mathrm{CH}), 129.3(\mathrm{CH}), 129.0(\mathrm{CH}), 128.5,128.0(\mathrm{CH}), 126.2,97.1(\mathrm{CH}), 91.8,81.7,61.8$ $\left(\mathrm{CH}_{2}\right)$, $54.4\left(\mathrm{CH}_{2}\right), 30.0\left(\mathrm{CH}_{2}\right), 25.1\left(\mathrm{CH}_{2}\right), 18.7\left(\mathrm{CH}_{2}\right)$ ppm; HRMS-CI $(\mathrm{m} / z)$ calcd for $\left[\mathrm{C}_{18} \mathrm{H}_{17} \mathrm{NO}_{3}+\mathrm{H}\right]^{+}:$296.1285; Found: 296.1287.

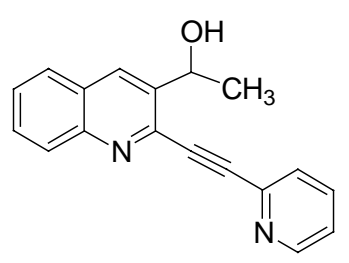

\section{1-(2-Pyridin-2-ylethynyl-quinolin-3-yl)-ethanol (2a)}

2-Pyridin-2-ylethynyl-quinoline-3-carbaldehyde (1a, $1.48 \mathrm{~g}, 5.73 \mathrm{mmol}, 1$ equiv) was diluted in dry THF (40.0 mL) under stirring and cooled at 0-5 ${ }^{\circ} \mathrm{C}$. After addition of methylmagnesium bromide $\left(3 \mathrm{M}\right.$ in $\mathrm{Et}_{2} \mathrm{O}, 2.5 \mathrm{~mL}$,

$7.45 \mathrm{mmol}, 1.3$ equiv), the solution became dark. After $30 \mathrm{~min}$ the reaction was quenched by addition of a saturated solution of $\mathrm{NH}_{4} \mathrm{Cl}$. Dilution in EtOAc and washing with water and brine followed by purification by flash chromatography on silica (EtOAc/cyclohexane, 4/1 then 100\% EtOAc) afforded 89\% yield of the alcohol 2a as an orange solid (1.40 g, $5.10 \mathrm{mmol}) . \mathrm{mp} 81-83$ ${ }^{\circ} \mathrm{C}$; $\mathrm{R}_{f}$ (cyclohexane/EtOAc, 1/2): 0.18; IR (neat) 3312, 3054, 2971, 2926, 1581, 1465, $1429 \mathrm{~cm}^{-1}$; ${ }^{1} \mathrm{H}$ NMR $\left(300 \mathrm{MHz}, \mathrm{CDCl}_{3}, \delta\right): 8.67$ (ddd, $\left.J=5.1 \mathrm{~Hz}, J=2.1 \mathrm{~Hz}, \mathrm{~J}=0.6 \mathrm{~Hz}, 1 \mathrm{H}\right), 8.39$ (s, $1 \mathrm{H}$ ), 8.12 (bd, $J=7.8 \mathrm{~Hz}, 1 \mathrm{H}), 7.85$ (bd, $J=8.1 \mathrm{~Hz}, 1 \mathrm{H}), 7.77-7.69$ (m, 3H), 7.57 (ddd, $J=8.1 \mathrm{~Hz}, J=$ 
$6.9 \mathrm{~Hz}, J=1.0 \mathrm{~Hz}, 1 \mathrm{H}), 7.32(\mathrm{ddd}, J=7.5 \mathrm{~Hz}, J=8.4 \mathrm{~Hz}, J=1.0 \mathrm{~Hz}, 1 \mathrm{H}), 5.66(\mathrm{q}, J=6.3 \mathrm{~Hz}$, 1H), $4.80(\mathrm{bs}, 1 \mathrm{H}), 1.71(\mathrm{~d}, J=6.3 \mathrm{~Hz}, 3 \mathrm{H}) ;{ }^{13} \mathrm{C} \mathrm{NMR}\left(75 \mathrm{MHz}, \mathrm{CDCl}_{3}, \delta\right): 149.8(\mathrm{CH}), 147.1$, 142.2, 141.3, 140.8, $136.3(\mathrm{CH}), 132.3(\mathrm{CH}), 129.5(\mathrm{CH}), 128.7(\mathrm{CH}), 127.9(\mathrm{CH}), 127.7,127.6$ $(\mathrm{CH}), 127.4(\mathrm{CH}), 123.5(\mathrm{CH}), 91.6,86.5,66.4(\mathrm{CH}), 24.4\left(\mathrm{CH}_{3}\right)$ ppm; HRMS-CI $(\mathrm{m} / \mathrm{z})$ calcd for $\left[\mathrm{C}_{18} \mathrm{H}_{14} \mathrm{~N}_{2} \mathrm{O}+\mathrm{H}\right]^{+}:$275.1184; Found: 275.1184 .

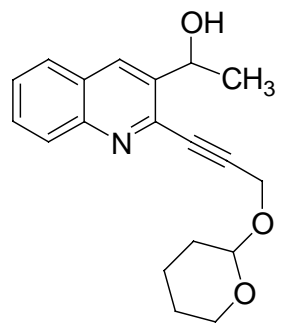

1-\{2-[3-(Tetrahydro-pyran-2-yloxy)-prop-1-ynyl]-quinolin-3-yl\}-ethanol (2b)

2-[3-(Tetrahydro-pyran-2-yloxy)-prop-1-ynyl]-quinoline-3-carbaldehyde (1b, $0.40 \mathrm{~g}, 1.35 \mathrm{mmol}, 1$ equiv) was diluted in dry THF (5 mL) under stirring and cooled at $-78{ }^{\circ} \mathrm{C}$. After addition of methylmagnesium bromide $\left(3 \mathrm{M}\right.$ in $\mathrm{Et}_{2} \mathrm{O}$, $1.0 \mathrm{~mL}, 2.97 \mathrm{mmol}, 2.2$ equiv), the solution if left at the same temperature for $5.5 \mathrm{~h}$ and quenched by addition of a saturated solution of $\mathrm{NH}_{4} \mathrm{Cl}$. Dilution in EtOAc and washing with a saturated solution of $\mathrm{NH}_{4} \mathrm{Cl}$ (twice) and brine (three times) followed by purification by flash chromatography on silica (EtOAc/cyclohexane, 6/4) afforded 64\% yield of the alcohol $\mathbf{2 b}$ as a light brown oil (0.27 g, $0.86 \mathrm{mmol}$ ). $\mathrm{R}_{f}$ (cyclohexane/EtOAc, 4/6): 0.48; IR (neat) 3353, 2941, 2851, 1618, 1595, 1557, 1490, 1343, 1120, 1060, $901 \mathrm{~cm}^{-1} ;{ }^{1} \mathrm{H}$ NMR (300 MHz, CDCl 3 , $): 8.27$ (s, 1H), $8.03(\mathrm{bd}, J=8.5 \mathrm{~Hz}, 1 \mathrm{H}), 7.76(\mathrm{bdd}, J=8.1 \mathrm{~Hz}, J=0.8 \mathrm{~Hz}, 1 \mathrm{H}), 7.66$ (ddd, $J=7.2 \mathrm{~Hz}$, $J=6.8 \mathrm{~Hz}, J=1.3 \mathrm{~Hz}, 1 \mathrm{H}), 7.50(\mathrm{ddd}, J=8.1 \mathrm{~Hz}, J=6.8 \mathrm{~Hz}, J=1.1 \mathrm{~Hz}, 1 \mathrm{H}), 5.42(\mathrm{q}, J=6.5$ $\mathrm{Hz}, 1 \mathrm{H}), 4.90(\mathrm{bs}, 1 \mathrm{H}), 4.56(\mathrm{~s}, 2 \mathrm{H}), 3.88(\mathrm{ddd}, J=8.5 \mathrm{~Hz}, J=8.1 \mathrm{~Hz}, J=2.8 \mathrm{~Hz}, 1 \mathrm{H}), 3.55(\mathrm{~m}$, $1 \mathrm{H}), 2.98(\mathrm{bs}, 1 \mathrm{H}), 1.85-1.52(\mathrm{~cm}, 9 \mathrm{H})$ including at $1.60(\mathrm{dd}, J=6.5 \mathrm{~Hz}, J=0.8 \mathrm{~Hz}) ;{ }^{13} \mathrm{C} \mathrm{NMR}$ (75 MHz, $\left.\mathrm{CDCl}_{3}, \delta\right): 147.5,141.5,140.8,132.5(\mathrm{CH}), 130.1(\mathrm{CH}), 129.2(\mathrm{CH}), 128.0(\mathrm{CH})$, 127.9, $127.8(\mathrm{CH}), 97.8(\mathrm{CH}), 90.7,83.9,67.5(\mathrm{CH}), 62.6\left(\mathrm{CH}_{2}\right), 55.2\left(\mathrm{CH}_{2}\right), 30.7\left(\mathrm{CH}_{2}\right), 25.7$ $\left(\mathrm{CH}_{2}\right), 24.6\left(\mathrm{CH}_{3}\right), 19.5\left(\mathrm{CH}_{2}\right)$ ppm; HRMS-CI $(\mathrm{m} / z)$ calcd for $\left[\mathrm{C}_{19} \mathrm{H}_{21} \mathrm{NO}_{3}+\mathrm{H}\right]^{+}: 312.1599$; Found: 312.1598 .

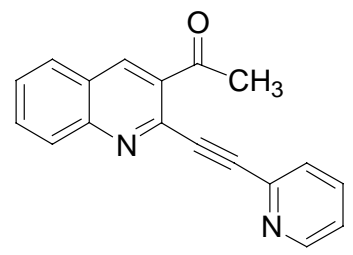

\section{1-(2-Pyridin-2-ylethynyl-quinolin-3-yl)-ethanone (3a)}

1-(2-Pyridin-2-ylethynyl-quinolin-3-yl)-ethanol (2a, $511 \mathrm{mg}, 1.86 \mathrm{mmol}$, 1 equiv) was diluted in dry $\mathrm{CH}_{2} \mathrm{Cl}_{2}(10.0 \mathrm{~mL})$ under stirring. Dess-Martin Periodinane (15\% wt, $5.81 \mathrm{~mL}, 2.79 \mathrm{mmol}, 1.5$ equiv) was then added at 
$0^{\circ} \mathrm{C}$ under argon. The solution was stirred $1 \mathrm{~h} 45$ at room temperature. The reaction was quenched by addition of a mixture of $\mathrm{Na}_{2} \mathrm{~S}_{2} \mathrm{O}_{3}$ and $\mathrm{NaHCO}_{3}$ solutions. The organic layer was washed with water then brine. Purification by flash chromatography on silica gel (EtOAc/cyclohexane, 2/1, $3 / 1$ then 5/1) afforded 75\% yield of compound 3a as a dark yellow solid (383 $\mathrm{mg}, 1.41 \mathrm{mmol}$ ). mp $111^{\circ} \mathrm{C} ; \mathrm{R}_{f}$ (cyclohexane/EtOAc 4/6): 0.35; IR (neat) 3053, 1670, 1579, 1546, 1428, 1222 , $988 \mathrm{~cm}^{-1} ;{ }^{1} \mathrm{H} \mathrm{NMR}\left(300 \mathrm{MHz}, \mathrm{CDCl}_{3}, \delta\right): 8.69(\mathrm{ddd}, J=5.1 \mathrm{~Hz}, J=2.1 \mathrm{~Hz}, J=0.6 \mathrm{~Hz}, 1 \mathrm{H})$, 8.60 (s, 1H), 8.17 (bd, $J=7.8 \mathrm{~Hz}, 1 \mathrm{H}), 7.93(\mathrm{bd}, J=8.1 \mathrm{~Hz}, 1 \mathrm{H}), 7.85$ (bdd, $J=7.5 \mathrm{~Hz}, J=1.8$ $\mathrm{Hz}, 1 \mathrm{H}), 7.79-7.71(\mathrm{~m}, 2 \mathrm{H}), 7.64(\mathrm{ddd}, J=8.1 \mathrm{~Hz}, J=6.9 \mathrm{~Hz}, J=1.0 \mathrm{~Hz}, 1 \mathrm{H}), 7.32(\mathrm{ddd}, J=7.5$ $\mathrm{Hz}, J=8.4 \mathrm{~Hz}, J=1.0 \mathrm{~Hz}, 1 \mathrm{H}), 2.96(\mathrm{~s}, 3 \mathrm{H}) ;{ }^{13} \mathrm{C} \mathrm{NMR}\left(75 \mathrm{MHz}, \mathrm{CDCl}_{3}, \delta\right): 198.4,150.2(\mathrm{CH})$, 148.6, 142.3, 139.5, $137.7(\mathrm{CH}), 136.1(\mathrm{CH}), 134.1,132.1(\mathrm{CH}), 129.1(\mathrm{CH}), 128.6(\mathrm{CH}), 128.2$ $(\mathrm{CH}), 127.8(\mathrm{CH}), 126.2,123.6(\mathrm{CH}), 92.1,87.4,29.8\left(\mathrm{CH}_{3}\right)$ ppm; HRMS-LSIMS $(\mathrm{m} / z)$ calcd for $\left[\mathrm{C}_{18} \mathrm{H}_{12} \mathrm{~N}_{2} \mathrm{O}+\mathrm{H}\right]^{+}: 273.1028$; Found: 273.1027 .

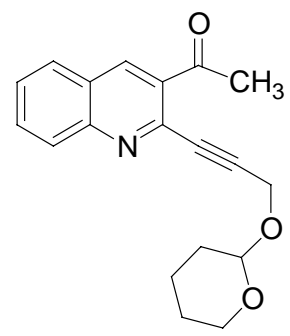

\section{1-\{2-[3-(Tetrahydro-pyran-2-yloxy)-prop-1-ynyl]-quinolin-3-yl\}-} ethanone (3b)

Same conditions as for 3a; using 1-\{2-[3-(Tetrahydro-pyran-2-yloxy)-prop-1ynyl]-quinolin-3-yl \}-ethanol (2b, $100 \mathrm{mg}, 0.32 \mathrm{mmol})$ we obtained $81 \%$ yield of compound $\mathbf{3 b}$ as a brown solid (80 mg, $0.26 \mathrm{mmol}$ ). $\mathrm{mp} 94-95{ }^{\circ} \mathrm{C} ; \mathrm{R}_{f}$ (cyclohexane/EtOAc, 4/6): 0.58; IR (neat) 2941, 1684, 1615, 1562, 1463, 1343, 1287, 1123, 1026 $\mathrm{cm}^{-1} ;{ }^{1} \mathrm{H}$ NMR $\left(300 \mathrm{MHz}, \mathrm{CDCl}_{3}, \delta\right): 8.43(\mathrm{~s}, 1 \mathrm{H}), 8.03(\mathrm{bd}, J=8.5 \mathrm{~Hz}, 1 \mathrm{H}), 7.80$ (bdd, $J=8.1$ $\mathrm{Hz}, J=0.8 \mathrm{~Hz}, 1 \mathrm{H}), 7.73(\mathrm{ddd}, J=8.3 \mathrm{~Hz}, J=7.2 \mathrm{~Hz}, J=1.3 \mathrm{~Hz}, 1 \mathrm{H}), 7.52(\mathrm{ddd}, J=7.2 \mathrm{~Hz}, J=$ $7.0 \mathrm{~Hz}, J=1.1 \mathrm{~Hz}, 1 \mathrm{H}), 4.91$ (appt, $J=3.1 \mathrm{~Hz}, 1 \mathrm{H}), 4.55$ (d, $J=1.88 \mathrm{~Hz}, 2 \mathrm{H}), 3.82$ (ddd, $J=8.5$ $\mathrm{Hz}, J=8.5 \mathrm{~Hz}, J=3.2 \mathrm{~Hz}, 1 \mathrm{H}), 3.55-3.48(\mathrm{~m}, 1 \mathrm{H}), 2.77(\mathrm{~s}, 3 \mathrm{H}), 1.81-1.47(\mathrm{~cm}, 6 \mathrm{H}) ;{ }^{13} \mathrm{C} \mathrm{NMR}$ (75 MHz, $\left.\mathrm{CDCl}_{3}, \delta\right):$ 199.0, 148.9, 140.0, $137.9(\mathrm{CH}), 134.4,132.4(\mathrm{CH}), 129.4(\mathrm{CH}), 129.0$ $(\mathrm{CH}), 128.5(\mathrm{CH}), 126.5,97.5(\mathrm{CH}), 91.0,85.3,62.4\left(\mathrm{CH}_{2}\right), 55.0\left(\mathrm{CH}_{2}\right), 30.6\left(\mathrm{CH}_{2}\right), 30.2\left(\mathrm{CH}_{3}\right)$, $25.7\left(\mathrm{CH}_{2}\right), 19.4\left(\mathrm{CH}_{2}\right)$ ppm; HRMS-CI $(\mathrm{m} / \mathrm{z})$ calcd for $\left[\mathrm{C}_{19} \mathrm{H}_{19} \mathrm{NO}_{3}+\mathrm{H}\right]^{+}:$310.1443; Found: 310.1440 . 


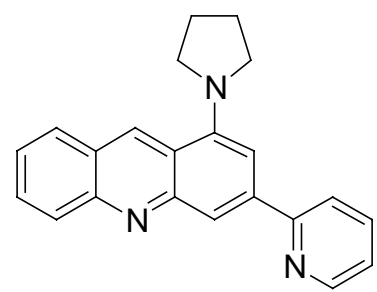

\section{3-Pyridin-2-yl-1-pyrrolidin-1-yl-acridine (4a)}

Following method A with pyrrolidine. The purification by flash chromatography on silica gel (eluant: cyclohexane/EtOAc, 1/1) afforded $46 \%$ yield of 3-Pyridin-2-yl-1-pyrrolidin-1-yl-acridine (4a, $55 \mathrm{mg}, 0.17$ mmol) as a red solid. Method B: 4a is obtained from 3a (85 $\mathrm{mg}, 0.31$ mmol) with a yield of $78 \%$ (80 mg, $0.25 \mathrm{mmol}$ ) after PTLC purification (cyclohexane/EtOAc, 4/6). Method C: $4 \mathbf{a}$ is obtained from $3 \mathbf{a}(50 \mathrm{mg}, 0.18 \mathrm{mmol})$ with a yield of $85 \%$ (51 mg, 0.16 mmol) after PTLC purification (cyclohexane/EtOAc, 4/6). $\mathrm{mp}$ 162-163.5 ${ }^{\circ} \mathrm{C} ; \quad \mathrm{R}_{f}$ (cyclohexane/EtOAc 4/6): 0.51; IR (neat) 2943, 2810, 1685, 1585, 1553, 1517, $1468 \mathrm{~cm}^{-1}$; ${ }^{1} \mathrm{H}$ NMR (300 MHz, $\mathrm{CDCl}_{3}, \delta$ ): 9.15 (s, 1H), 8.76 (bd, $\left.J=4.5 \mathrm{~Hz}, 1 \mathrm{H}\right), 8.27$ (s, 1H), 8.19 (bd, $J=$ $8.6 \mathrm{~Hz}, 1 \mathrm{H}), 8.01$ (appt, $J=8.1 \mathrm{~Hz}, 2 \mathrm{H}), 7.84-7.76(\mathrm{~m}, 2 \mathrm{H}), 7.74(\mathrm{~d}, J=1.5 \mathrm{~Hz}, 1 \mathrm{H}), 7.50$ (ddd, $J$ $=8.1 \mathrm{~Hz}, J=6.9 \mathrm{~Hz}, J=1.0 \mathrm{~Hz}, 1 \mathrm{H}), 7.29(\mathrm{ddd}, J=7.5 \mathrm{~Hz}, J=8.4 \mathrm{~Hz}, J=1.0 \mathrm{~Hz}, 1 \mathrm{H}), 3.63(\mathrm{~m}$, 4H), $2.10(\mathrm{~m}, 4 \mathrm{H}) ;{ }^{13} \mathrm{C} \mathrm{NMR}\left(75 \mathrm{MHz}, \mathrm{CDCl}_{3}, \delta\right.$ ): 157.3, 150.6, $149.5(\mathrm{CH}), 148.7,148.4,141.4$, $136.8(\mathrm{CH}), 133.9(\mathrm{CH}), 130.4(\mathrm{CH}), 128.7(\mathrm{CH}), 125.3,125.2(\mathrm{CH}), 122.5(\mathrm{CH}), 122.4,121.5$ $(\mathrm{CH}), 119.3(\mathrm{CH}), 107.5(\mathrm{CH}), 53.0\left(\mathrm{CH}_{2}\right), 25.2\left(\mathrm{CH}_{2}\right)$ ppm; HRMS-CI $(\mathrm{m} / z)$ calcd for $\left[\mathrm{C}_{22} \mathrm{H}_{19} \mathrm{~N}_{3}+\mathrm{H}\right]^{+}:$326.1657; Found: 326.1657 .

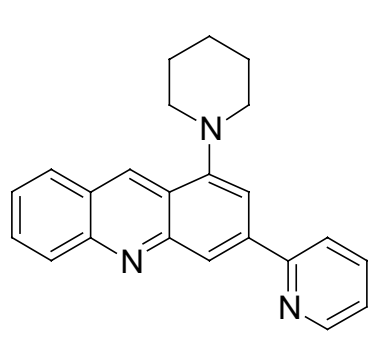

\section{1-Piperidin-1-yl-3-pyridin-2-yl-acridine (4b)}

Method A with piperidine afforded, after purification by PTLC silica plate (eluant: cyclohexane/EtOAc, 1/2), 11\% yield of 1-Piperidin-1-yl-3pyridin-2-yl-acridine $(\mathbf{4 b}, 14 \mathrm{mg}, 0.041 \mathrm{mmol})$ as a dark yellow solid.

Method B afforded 50\% yield of $\mathbf{4 b}$ as seen by ${ }^{1} \mathrm{H}$ NMR of the crude, the other $50 \%$ being the enamine intermediate (Characteristic ${ }^{1} \mathrm{H}$ enamine $\mathrm{CH}_{2}$ peak at $6.36 \mathrm{ppm}$ integrating for two protons compared with the ${ }^{1} \mathrm{H}$ at $9.08 \mathrm{ppm}$ characteristic of the acridine's peaks and integrating for one proton). With Method $\mathbf{C}$ only traces ( $5 \%)$ of $\mathbf{4 b}$ was seen by ${ }^{1} \mathrm{H}$ NMR. mp 155-156 ${ }^{\circ} \mathrm{C} ; \mathrm{R}_{f}$ (cyclohexane/EtOAc 4/6): 0.60; IR (neat) 2925, 1544, 1517, 1445, 1376, $930 \mathrm{~cm}^{-1} ;{ }^{1} \mathrm{H}$ NMR (300 MHz, $\left.\mathrm{CDCl}_{3}, \delta\right): 9.08$ (s, 1H), 8.78 (ddd, $J=5.1 \mathrm{~Hz}, J=2.1 \mathrm{~Hz}, J$ $=0.6 \mathrm{~Hz}, 1 \mathrm{H}), 8.41(\mathrm{bs}, 1 \mathrm{H}), 8.22(\mathrm{bd}, J=7.5 \mathrm{~Hz}, 1 \mathrm{H}), 8.05(\mathrm{bd}, J=7.2 \mathrm{~Hz}, 1 \mathrm{H}), 8.03(\mathrm{bd}, J=$ $8.1 \mathrm{~Hz}, 1 \mathrm{H}), 7.94(\mathrm{~d}, J=1.5 \mathrm{~Hz}, 1 \mathrm{H}), 7.83(\mathrm{td}, J=7.5 \mathrm{~Hz}, J=1.8 \mathrm{~Hz}, 1 \mathrm{H}), 7.79$ (td, $J=9.0 \mathrm{~Hz}, J$ $=1.5 \mathrm{~Hz}, 1 \mathrm{H}), 7.54(\mathrm{ddd}, J=8.1 \mathrm{~Hz}, J=6.9 \mathrm{~Hz}, \mathrm{~J}=1.0 \mathrm{~Hz}, 1 \mathrm{H}), 7.30(\mathrm{ddd}, J=7.5 \mathrm{~Hz}, J=8.4$ $\mathrm{Hz}, J=1.0 \mathrm{~Hz}, 1 \mathrm{H}), 3.25$ (bs, 4H), 1.95 (m, 4H), 1.74 (bs, 2H); ${ }^{13} \mathrm{C} \mathrm{NMR} \mathrm{(75} \mathrm{MHz,} \mathrm{CDCl}_{3}$, 


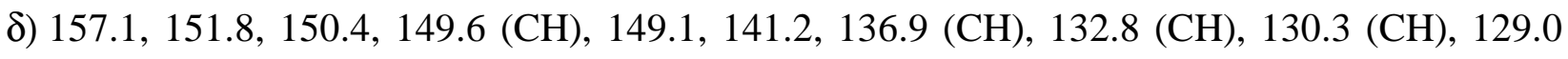
$(\mathrm{CH}), 128.8(\mathrm{CH}), 126.2,125.5(\mathrm{CH}), 123.3,122.6(\mathrm{CH}), 122.0(\mathrm{CH}), 121.4(\mathrm{CH}), 112.4(\mathrm{CH})$, $54.8\left(\mathrm{CH}_{2}\right), 26.6\left(\mathrm{CH}_{2}\right), 24.6\left(\mathrm{CH}_{2}\right)$ ppm; HRMS-LSIMS $(\mathrm{m} / z)$ calcd for $\left[\mathrm{C}_{23} \mathrm{H}_{21} \mathrm{~N}_{3}+\mathrm{H}\right]^{+}$: 340.1814; Found: 340.1811.

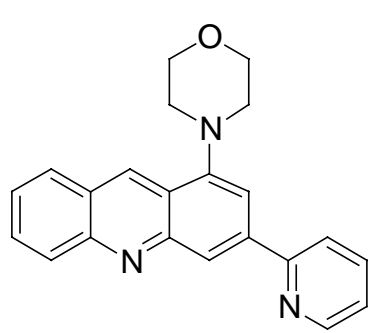

\section{1-Morpholin-4-yl-3-pyridin-2-yl-acridine (4c)}

Using Method D with $50 \mathrm{mg}$ of $\mathbf{3 a}(0.18 \mathrm{mmol})$ we obtained $\mathbf{4 c}$, as a yellow solid, with a yield of $81 \%(51 \mathrm{mg}, 0.15 \mathrm{mmol})$ after PTLC purification (cyclohexane/EtOAc, 4/6). $\quad \begin{array}{llllll} & \mathrm{mp} & 205 & { }^{\circ} \mathrm{C} & \text { dec.; } & \mathrm{R}_{f}\end{array}$ (cyclohexane/EtOAc 4/6): 0.42; IR (neat) 3047, 2962, 2931, 2857, 2824, 1692, 1626, 1586, 1537, 1519, 1260, 1191, $860 \mathrm{~cm}^{-1} ;{ }^{1} \mathrm{H}$ NMR (300 MHz, $\mathrm{CDCl}_{3}, \delta$ ): 9.09 (s, $1 \mathrm{H}), 8.78$ (bdd, $J=4.8 \mathrm{~Hz}, J=0.7 \mathrm{~Hz}, 1 \mathrm{H}$ ), 8.46 (bs, $1 \mathrm{H}$ ), 8.22 (bd, $J=8.9 \mathrm{~Hz}, 1 \mathrm{H}$ ), 8.06-8.02 $(\mathrm{m}, 2 \mathrm{H}), 8.00(\mathrm{~d}, J=1.3 \mathrm{~Hz}, 1 \mathrm{H}), 7.84(\mathrm{dd}, J=7.5 \mathrm{~Hz}, J=1.9 \mathrm{~Hz}, 1 \mathrm{H}), 7.78(\mathrm{dd}, J=9.0 \mathrm{~Hz}, J=$ $1.3 \mathrm{~Hz}, 1 \mathrm{H}$ ), 7.55 (ddd, $J=8.1 \mathrm{~Hz}, J=6.6 \mathrm{~Hz}, \mathrm{~J}=0.9 \mathrm{~Hz}, 1 \mathrm{H}$ ), 7.31 (ddd, $J=7.5 \mathrm{~Hz}, J=4.7 \mathrm{~Hz}$, $J=0.9 \mathrm{~Hz}, 1 \mathrm{H}), 4.08(\mathrm{~m}, 4 \mathrm{H}), 3.30(\mathrm{~m}, 4 \mathrm{H}) ;{ }^{13} \mathrm{C} \mathrm{NMR}\left(75 \mathrm{MHz}, \mathrm{CDCl}_{3}, \delta\right)$ 156.8, 150.4, 150.1, 149.7, 149.2, 141.1, $137.0(\mathrm{CH}), 132.3(\mathrm{CH}), 130.6(\mathrm{CH}), 129.1(\mathrm{CH}), 128.7(\mathrm{CH}), 126.2,125.8$ $(\mathrm{CH}), 122.9,122.79(\mathrm{CH}), 122.76,121.4(\mathrm{CH}), 112.8(\mathrm{CH}), 67.4\left(\mathrm{CH}_{2}\right), 53.7\left(\mathrm{CH}_{2}\right)$ ppm; HRMS-CI $(m / z)$ calcd for $\left[\mathrm{C}_{22} \mathrm{H}_{19} \mathrm{~N}_{3} \mathrm{O}+\mathrm{H}\right]^{+}:$342.1606; Found: 342.1604 .

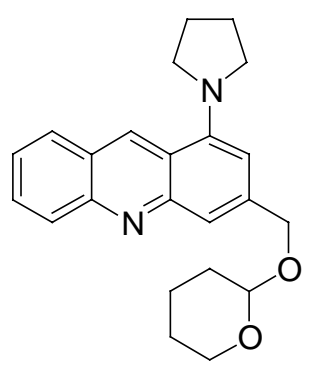

\section{1-Pyrrolidin-1-yl-3-(tetrahydro-pyran-2-yloxymethyl)-acridine (4d)}

Using Method $\mathbf{C}$ with pyrrolidine and $70 \mathrm{mg}$ of $\mathbf{3 b}(0.23 \mathrm{mmol})$ we obtained 4d, as a reddish gum, with a yield of $84 \%$ (69 $\mathrm{mg}, 0.19 \mathrm{mmol}$ ) after PTLC purification (cyclohexane/EtOAc, 4/6). $\mathrm{R}_{f}$ (cyclohexane/EtOAc 4/6): 0.60; IR (neat) 2921, 2848, 1557, 1454, 1336, 1116, $983 \mathrm{~cm}^{-1} ;{ }^{1} \mathrm{H}$ NMR $(300 \mathrm{MHz}$, $\mathrm{CDCl}_{3}, \delta$ ): 9.10 (s, 1H), 8.17 (bd, $\left.J=8.85 \mathrm{~Hz}, 1 \mathrm{H}\right), 7.98$ (bd, $J=8.30 \mathrm{~Hz}$, 1H), 7.76 (bs, 2H), 7.48 (ddd, $J=7.3 \mathrm{~Hz}, J=7.0 \mathrm{~Hz}, J=1.1 \mathrm{~Hz}, 1 \mathrm{H}), 6.81(\mathrm{~s}, 1 \mathrm{H}), 4.98$ (d, $J=$ $13.0 \mathrm{~Hz}, 1 \mathrm{H}), 4.82$ (appt, $J=3.4 \mathrm{~Hz}, 1 \mathrm{H}), 4.70$ (d, $J=13.0 \mathrm{~Hz}, 1 \mathrm{H}), 3.99$ (ddd, $J=8.1 \mathrm{~Hz}, J=$ $7.9 \mathrm{~Hz}, J=3.4 \mathrm{~Hz}, 1 \mathrm{H}), 3.62-3.50(\mathrm{~m}, 5 \mathrm{H}), 2.09(\mathrm{~m}, 4 \mathrm{H}), 1.96-1.54(\mathrm{~cm}, 6 \mathrm{H}) ;{ }^{13} \mathrm{C}$ NMR $(75$ $\left.\mathrm{MHz}, \mathrm{CDCl}_{3}, \delta\right) 150.5,148.5,148.1,141.3,133.8(\mathrm{CH}), 130.3(\mathrm{CH}), 128.72(\mathrm{CH}), 128.66(\mathrm{CH})$, 125.0, $124.9(\mathrm{CH}), 121.8,119.1(\mathrm{CH}), 108.0(\mathrm{CH}), 98.0(\mathrm{CH}), 69.1\left(\mathrm{CH}_{2}\right), 62.2\left(\mathrm{CH}_{2}\right), 52.9$ 
$\left(\mathrm{CH}_{2}\right), 30.6\left(\mathrm{CH}_{2}\right), 25.5\left(\mathrm{CH}_{2}\right), 25.2\left(\mathrm{CH}_{2}\right), 19.3\left(\mathrm{CH}_{2}\right)$ ppm; HRMS-CI $(\mathrm{m} / \mathrm{z})$ calcd for $\left[\mathrm{C}_{23} \mathrm{H}_{26} \mathrm{~N}_{2} \mathrm{O}_{2}+\mathrm{H}\right]^{+}:$363.2072; Found: 363.2073.

Enamines are quite sensitive functionalities, which explain why only ${ }^{1} \mathrm{H}$ NMR spectrum was performed for compound $\mathbf{4 e}$.

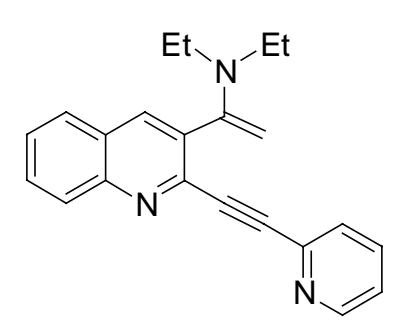

Diethyl-[1-(2-pyridin-2-ylethynyl-quinolin-3-yl)-vinyl]-amine (4e)

Using Method B or Method C with diethylamine and $100 \mathrm{mg}$ of $\mathbf{3 b}$ (0.36 mmol) we obtained a total conversion into 4e. $\mathrm{R}_{f}$ (cyclohexane/EtOAc 4/6): 0.40; IR (neat) 2926, 1686, 1583, 1378, 1245, $993 \mathrm{~cm}^{-1} ;{ }^{1} \mathrm{H}$ NMR $\left(300 \mathrm{MHz}, \mathrm{CDCl}_{3}, \delta\right):{ }^{1} \mathrm{H} \mathrm{NMR}\left(300 \mathrm{MHz}, \mathrm{CDCl}_{3}\right.$, ठ): 8.64 (bs, 2H), 8.05 (bs, 2H), 7.6-7.5 (bs, 3H), 7.42 (appt, $J=7.3 \mathrm{~Hz}, 1 \mathrm{H}), 7.06$ (bd, $J=8.3$ $\mathrm{Hz}, 1 \mathrm{H}), 6.30$ (s, 2H), 3.25 (m, 4H), $1.19(\mathrm{~m}, 6 \mathrm{H})$. 